\title{
ÉCOLES ET VIOLENCES : LES LEÇONS BRÉSILIENNES Benjamin Moignard
}

\author{
ERES | « Enfances \& Psy » \\ 2009/4 n $45 \mid$ pages 109 à 118 \\ ISSN 1286-5559 \\ ISBN 9782749211473
}

Article disponible en ligne à l'adresse :

https://www.cairn.info/revue-enfances-et-psy-2009-4-page-109.htm

\section{Pour citer cet article :}

Benjamin Moignard, «Écoles et violences : les leçons brésiliennes », Enfances \& Psy 2009/4 (n 45), p. 109-118.

DOI 10.3917/ep.045.0109

Distribution électronique Cairn.info pour ERES.

(C) ERES. Tous droits réservés pour tous pays.

La reproduction ou représentation de cet article, notamment par photocopie, n'est autorisée que dans les limites des conditions générales d'utilisation du site ou, le cas échéant, des conditions générales de la licence souscrite par votre établissement. Toute autre reproduction ou représentation, en tout ou partie, sous quelque forme et de quelque manière que ce soit, est interdite sauf accord préalable et écrit de l'éditeur, en dehors des cas prévus par la législation en vigueur en France. Il est précisé que son stockage dans une base de données est également interdit. 


\section{Benjamin Moignard Écoles et violences : les leçons brésiliennes}

\section{VIOLENCE ET PAUVRETÉ : DES LIENS D'ÉVIDENCE À REPENSER}

Un certain nombre de travaux menés en France ou dans d'autres pays européens ont clairement démontré que la violence à l'école ${ }^{1}$ est liée à une sociologie de l'exclusion sociale. En d'autres termes, le niveau de violence mesuré dans les différents pays est généralement plus important dans les établissements qui accueillent des publics dits « défavorisés » que dans les autres. Si l'on prend le cas de la France, les dernières enquêtes de victimation ${ }^{2}$, produites sous l'égide de l'observatoire européen de la violence scolaire en 2003 dans le secondaire, montrent clairement que si le niveau de la violence à l'école est relativement stable depuis 1993 sur l'ensemble du territoire, les établissements classés en ZEP sont plus touchés que les autres. Cette situation tend à s'accentuer avec les années : les inégalités sociales face à la violence scolaire se renforcent donc en France. On observe d'ailleurs, dans des proportions moindres cependant, la même tendance dans de nombreux pays européens ${ }^{3}$. Les recherches anglo-saxonnes, dont beaucoup sont organisées sur la définition des facteurs de risques liés à la violence, pointent elles aussi le milieu socio-économique des élèves comme un élément déterminant du niveau de violence dans les établissements scolaires. Ces résultats pourraient donc conforter le discours d'un certain nombre d'acteurs scolaires sur le risque d'une « contamination » de l'école par la violence supposée des quartiers. Plusieurs recherches accréditent d'ailleurs cette idée d'une violence intrusive, alimentée par le quartier, liée à la double pression de la démocratisation scolaire et de l'aggravation de
Benjamin Moignard est maître

de conférences en sociologie

à l'université de Paris 12,

membre du CIRCEFT

et de l'Observatoire international

de la violence à l'école.
1. Ce terme est envisagé à partir de son acception large : la violence, à l'école, qualifie à la fois les faits pénalement répréhensibles et ceux qui sont perçus par les acteurs de l'école comme des violences. Il ne s'agit pas de dire que toutes les manifestations déviantes, ou qualifiées comme telles selon l'univers normatif de l'école, sont des violences, mais que leur répétition peut aboutir à la perception d'une violence quotidienne et usante pour les différents acteurs de l'école, entrânant une détérioration durable du climat scolaire, indicateur incontournable de mesure du niveau de violence à l'école. Cf. à ce sujet É. Debarbieux, La violence en milieu scolaire. Tome 2 : Le désordre des choses, 1999, Paris, ESF. 
2. Ces enquêtes visent à contourner le fameux problème du « chiffre noir » de la délinquance : comment mesurer quelque chose qui est interdit ou répréhensible? Si

l'on ne considère

que les chiffres des administrations chargées de prendre en charge le phénomène (école, police, justice), alors c'est l'activité de ces services qui est mesurée, plutôt que la réalité sociale du phénomène considéré. Ces « enquêtes de victimation » visent à interroger des élèves, échantillonnés de façon à représenter la population d'un pays, d'une région, d'une ville, sur leur perception de la violence et les actes dont ils ont pu être victimes à l'école. Il ne s'agit plus alors de s'intéresser seulement aux élèves qui commettent des violences ou aux faits que l'administration traite, mais bien de considérer l'ensemble d'une population scolaire pour mesurer ce à quoi elle est confrontée.

3. Cf. en particulier à ce sujet le numéro spécial de l'International Journal on Violence and Schools «Un état de la recherche européenne sur la violence à l'école »,

9 septembre 2009. la violence dans l'environnement immédiat de l'école. La question n'est alors plus scolaire, elle devient la résurgence d'une problématique urbaine qui dépasse largement le cadre de l'école. C'est ainsi l'expérience du quartier qui développerait, en particulier chez les élèves issus des quartiers populaires, des comportements antiscolaires, voire une incapacité à se soumettre aux exigences scolaires, susceptibles d'alimenter des comportements violents. L'opposition entre les normes de la rue et celles de l'école deviendrait trop forte pour qu'elles puissent cohabiter (Ballion, 2000 ; Ozbolt et Peyrat, 2007).

Mais ce type d'approche a, à notre sens, le défaut de trop facilement amalgamer l'origine sociale des élèves et une supposée inclination à la violence qui suffirait à expliquer les difficultés rencontrées dans certains établissements populaires. Il ne s'agit pas de minorer le poids du quartier dans la structuration possible de la violence à l'école en milieu populaire, mais de chercher à déconstruire une approche qui pose, en termes d'évidence, des représentations et des réalités socialement construites. Même si le combat contre l'amalgame violence-pauvreté est devenu un lieu commun de la sociologie, il semble pourtant nécessaire de poursuivre l'argumentaire. Et la comparaison internationale sur l'objet « violence à l'école » est particulièrement précieuse en ce sens. En effet, si la violence à l'école en France, et plus largement en Europe et en Amérique du nord, semble bien être une violence de l'exclusion sociale, les résultats d'enquêtes de victimation comparables dans des pays au niveau de développement économique moins avancé offrent de nouvelles perspectives d'analyse. Ces enquêtes par questionnaire ont révélé par exemple des niveaux de violence à l'école plus faibles au Brésil qu'en France, y compris dans des quartiers soumis à un environnement beaucoup plus dur. Si l'on compare par exemple les victimations déclarées entre les deux pays, les chiffres sont clairs : si 24,6 \% des élèves interrogés en France disent avoir subi des coups au collège et $75 \%$ avoir été insultés, les taux chutent respectivement à $4,8 \%$ et $60 \%$ au Brésil ; les écoliers français sont $23 \%$ à se dire victimes du racisme dans l'établissement contre $9 \%$ des Brésiliens, et la présence d'extorsion est signalée par $37 \%$ des élèves en France contre $29 \%$ au Brésil (Debarbieux, 2006b, p. 184). On retrouve ces différences si l'on compare les relations entre les élèves et les professeurs : près de $25 \%$ des adolescents français les jugent mauvaises ou pas très bonnes contre seulement $11,5 \%$ des jeunes Brésiliens. Signalons également que « la perception de la violence chez les élèves brésiliens de 12 à 16 ans est nettement moins forte que pour leurs homologues français. En effet $31,1 \%$ des élèves de l'échantillon français de référence contre $12,4 \%$ des élèves brési- 
liens estiment la violence très présente dans leur établissement scolaire [modalités «énormément » et « beaucoup »]. [...] Ce sont en fait toutes les dimensions du climat scolaire qui sont meilleures d'après les élèves brésiliens » (Debarbieux, 2006a, p. 23). Cette situation n'est d'ailleurs pas particulière au Brésil puisque Debarbieux constate une tendance similaire dans des pays beaucoup plus pauvres comme la République de Djibouti ou le Burkina Faso, ce qui l'incite à parler d'un «paradoxe » de la violence à l'école, plus forte dans les pays riches, et qui remet donc en cause les modèles par facteurs de risques liés à la pauvreté et à l'exclusion sociale. D'autres exemples pourraient encore être présentés qui vont tous dans le même sens : le niveau socio-économique ne suffit pas à expliquer la violence à l'école.

Mais lorsque l'on a mesuré ces différences, encore faut-il essayer de les comprendre. C'est le sens de l'enquête ethnographique que nous avons menée en France et au Brésil entre 2002 et 2006 4 Sur la base des enquêtes quantitatives produites par l'observatoire, nous avons cherché à saisir à la fois la réalité sociale du quartier et la manière avec laquelle le collège est confronté de l'intérieur à un certain nombre de conduites déviantes et délinquantes. Il s'agit donc d'interroger la façon dont l'école interagit sur le plan de la construction des activités déviantes et délinquantes avec son environnement. La forte disparité constatée entre les réalités sociales françaises et brésiliennes va à l'encontre des idées reçues sur le lien entre un environnement violent et une école sous tension et révèle le rôle central de l'école et de ses modalités d'organisation dans la fabrication scolaire de la violence à l'école. La violence à l'école n'est donc pas qu'une affaire de milieu social ; elle est aussi, et peut-être d'abord, à l'échelle internationale, une question d'environnement scolaire.

\section{UN COLLÈGE QUI PROTÈGE}

Le Brésil a largement rattrapé son retard en matière d'éducation ces dix dernières années, en particulier à l'égard de l'école primaire et du collège où le taux de scolarisation atteint aujourd'hui celui des principaux pays développés, et ce même si de nombreux problèmes se posent encore (Ireland et al., 2007).

Le collège Paolo Freire est situé dans la partie basse de la favela qui le domine de toute part. Tous les élèves scolarisés vivent dans la favela de Rocinha alors que l'immense majorité des enseignants habite plutôt des immeubles récents situés dans des quartiers plus résidentiels. Les élèves arborent en guise d'uniforme un tee-shirt à l'effigie du collège et de la municipalité qui le finance. L'architecture de l'établissement est relativement simple, l'en-
4. Recherche appuyée et financée

par l'observatoire européen de

la violence scolaire,

l'université de Bordeaux II

et l'Unesco Brésil.

Menée en 2003 et 2005 ,

l'enquête française a porté sur un quartier populaire de la région parisienne particulièrement ségrégué. 35 entretiens ont été menés avec des collégiens, 8 auprès de jeunes adultes, et 15 avec des membres de l'équipe pédagogique de l'établissement situé au cœur du quartier. Au Brésil, l'enquête s'est organisée en 2004 à partir d'une période d'observation directe de huit mois menée dans un collège de la Rocinha, favela de Rio de Janeiro qui compte parmi les plus grandes d'Amérique latine. Cette favela est historiquement confrontée à d'importants problèmes de délinquance juvénile liée en particulier au narcotrafic qui occupe une place centrale dans la vie de la communauté locale.

Une fois cette entrée dans le collège assurée, j'ai pu tisser des relations étroites, par l'intermédiaire d'un informateur privilégié, avec un groupe d'adolescents impliqués dans le trafic de drogue local et qui restaient, pour une majorité d'entre eux, scolarisés. Un journal de bord a recensé les temps d'observation directe menée dans le quartier et dans le collège de chacun des deux terrains investis : 121 jours en France et 104 au Brésil. 
.......

Pour plus de détails sur le cadre méthodologique qui structure cette recherche et les difficultés rencontrées dans sa mise en place, cf. B. Moignard, L'école et la rue: fabriques de délinquance, Paris, PUF, 2008.

5. Adolescents qui participent à l'organisation du trafic de cocaïne dans la favela. semble formant un bloc composé de trois étages. Le rez-de-chaussée abrite une cour d'une centaine de mètres carrés et une cantine d'une capacité de deux cents places dans laquelle mangent près de six cents élèves chaque jour, répartis en différents services. Les salles de classe se distribuent le long d'un grand couloir au premier et au deuxième étage alors qu' une pièce est réservée aux professeurs et qu'un petit local sert de salle d'activités aux deux animateurs embauchés à plein temps dans l'établissement. Les bureaux de l'administration et de la direction font face aux salles de classe, situées de l'autre côté de la première moitié du couloir du premier étage. Une salle équipée en informatique est également accessible à certains horaires pour les élèves, le troisième étage abrite un gymnase. L'établissement est ceint par des grillages qui jouxtent un conduit par lequel sont évacués à l'air libre une partie des eaux usées et des déchets de la favela. Les élèves entrent et sortent à leur guise dans la cour alors que l'accès au premier étage est régulé par un surveillant. Dans l'ensemble, l'ambiance dans l'établissement est relativement sereine et les enseignants comme les élèves y font état d'une violence très réduite. L'école semble même considérée par de nombreux collégiens comme un espace de protection face à la violence extérieure. Les membres du trafico ${ }^{5}$ qui sont scolarisés vont d'ailleurs dans le même sens : l'un d'entre eux nous dit par exemple que le collège lui «permet de souffler », un autre adolescent présente l'établissement comme un « espace de paix ». L'école et les professeurs sont donc largement appréciés et la possibilité «d'accéder aux savoirs » est toujours considérée comme une «chance ». Nous n'avons pas constaté en effet de ressentiment particulier à l'égard du monde scolaire en général et des enseignants en particulier. L'observation de l'établissement pendant plusieurs mois confirme par ailleurs le discours des élèves : pendant toute la durée de notre séjour nous n'avons comptabilisé qu'une seule bagarre, l'ambiance générale est résolument bonne et l'agressivité entre les élèves ou entre élèves et professeurs est considérablement plus réduite que ce que nous avons pu observer en France (Moignard, 2007).

\section{UNE COMMUNAUTÉ DANS LE COLLÈGE, UN COLLÈGE DE LA COMMUNAUTÉ}

Nous savons que le climat scolaire est un des éléments décisifs de la diminution ou au contraire de l'augmentation de la violence à l'école, et il semble que l'ouverture de l'établissement à son environnement, l'implication des enseignants comme la participation des élèves à des projets menés au sein de l'établissement favorisent nettement son amélioration (Benbenisthy et Astor, 2005 ; Blaya, 2006 ; Gottfredson, 2001). Le Brésil est un bon exemple 
de cette conception d'une école ouverte, tournée vers la communauté qui entoure l'école, très éloignée du modèle républicain d'une école sanctuarisée que nous connaissons en France (Ireland et al., 2007). Dans le collège Paolo Freire de la Rocinha, des organisations internationales et des associations locales proposent tout au long de l'année aux élèves et aux familles des espaces ressources ou des activités dont un grand nombre sont conduites au sein même du collège. Elles sont organisées pendant la journée, mais aussi le soir et le week-end puisque l'établissement reste souvent ouvert ${ }^{6}$. Nous avons répertorié, sans viser l'exhaustivité, pas moins de dix-sept projets différents conduits par des ONG locales ou internationales dans l'enceinte de l'établissement. Les enseignants et la direction du collège sont impliqués directement dans la gestion de plus de la moitié d'entre eux, trouvant là l'occasion d'arrondir des fins de mois difficiles et signifiant aussi une forme d'engagement important auprès des élèves.

Il apparaît par ailleurs que les activités délinquantes et déviantes conduites dans l'enceinte de l'établissement sont particulièrement réduites - celles qui ont cours dans la rue n'ayant que peu de prise sur le collège. Si un certain nombre d'élèves scolarisés sont membres de gangs liés au trafic, il semble qu'ils adaptent leur conduite à l'école. Le collège est en fait assimilé à un espace protecteur et à protéger, il constitue clairement un point central de la vie de la communidade 7 , une référence partagée par les élèves, leurs parents et leurs voisins. Joca, chef d'un de ces groupes, avec qui nous nous sommes entretenus pendant près d'une année, nous dira ainsi que le collège est « intouchable » tandis que d'autres adolescents évoqueront l'établissement comme un « espace à part », un « lieu de vie qui appartient à tous » ou «l'un des meilleurs endroits de la favela » (Moignard, 2008). En d'autres termes, la politique de l'établissement comme les pratiques sociales dans la favela sont clairement orientées vers une démarche communautaire - non pas entendue au sens de « communautariste » comme on a trop souvent tendance à le réduire en France, mais plutôt comme « d'une communauté de vie »- qui vise à associer les familles, les élèves, les enseignants et les travailleurs sociaux dans un projet d'éducation et de socialisation globale dont l'école est le principal terrain à défaut d'être le seul instigateur. Le collège est au centre des actions de socialisation et d'éducation des élèves mais aussi de leur famille, tout à la fois relais, médiateur, éducateur, animateur, professeur, les enseignants combinant une pluralité de rôles et de fonctions qui ne garantissent pas toujours l'efficacité de l'action. Reste que si la question de la violence à l'école semble avoir été plutôt bien résolue, celle de la qualité des apprentissages se pose, comme nous le verrons.

\section{L'établissement scolaire, lieu de vie incontournable}

L'activité au collège n'est pas restreinte aux seuls exercices scolastiques, mais accueille aussi de nombreux intervenants et un certain nombre d'activités qui élargissent considérablement son champ d'action. Dans le discours des élèves, le collège est ainsi tout autant désigné comme un espace d'apprentissage du savoir scolaire académique que comme un lieu incontournable pour les loisirs et les pratiques socioculturelles au sens large. Si d'autres structures existent parallèlement dans la favela, c'est l'établissement scolaire qui est désigné comme le premier espace de ressources pour les activités extrascolaires. La perception du collège comme un espace protégé de la pression du narcotrafic revient également largement dans les raisons avancées pour le désigner comme un lieu de vie incontournable.

\section{Les élèves ont cours soit le matin, soit l'après-midi. Les activités socioculturelles proposées et auxquelles les élèves participent en très grand nombre contribuent cependant}




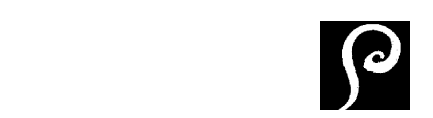

La violence à l'école

\begin{abstract}
Il serait pourtant réducteur d'attribuer tout le mérite du bon climat scolaire à l'ouverture de l'établissement sur la communauté ou à la seule implication des enseignants. La recherche internationale sur la question a largement pointé la pluralité d'éléments à prendre en compte pour comprendre la construction de la violence à l'école et du climat scolaire. L'ouverture communautaire serait-elle l'assurance d'une tranquillité scolaire à laquelle il serait légitime d'aspirer dans nos sociétés démocratiques ? S'agit-il là d'un élément décisif du maintien de la paix scolaire ? Pas si simple. Car s'il ne faut pas douter que le dynamisme et les pratiques locales favorisent la construction d'un climat serein et la diminution des conduites délinquantes dans le collège, il faut bien comprendre que cette tranquillité est aussi possible parce qu'elle est, selon des modalités particulières, acceptée par les tenants de l'ordre social dans la favela : les trafiquants. En d'autres termes, le collège, qui constitue un élément central de la vie de la communidade, peut aussi devenir un outil particulièrement efficace d'oppression paternaliste.
\end{abstract}

\title{
LE COLLÈGE COMME OUTIL DE L'OPPRESSION PATERNALISTE
}

Difficile effectivement de comprendre comment les chefs du trafic qui exercent sur la communauté une pression quotidienne tolèrent la mise en place d'un collège qui occupe une place centrale dans la vie de la communauté (Misse, 2008). L'histoire brésilienne et la longue dictature militaire qu'a traversée le pays ont été propices au développement d'une forme de paternalisme que l'on retrouve à tous les niveaux de la société. Christian Geffray rappelle ainsi que le « maître » a toujours les deux mains occupées : l'une sur le cœur pour s'assurer de «l'amour du dominé », l'autre sur le fusil pour faire parler la poudre si la loi de l'oppresseur n'est pas respectée (Geffray, 1995). Dans les favelas où le trafic de cocaïne occupe une place centrale dans l'activité locale, le dono 8 impose ses règles et devient le garant de l'ordre social d'une communidade abandonnée par un État qui la délaisse quand il ne la pourchasse pas (Rio Caldeira, 2002). Mais il ne suffit pas de porter des coups ou d'assurer la tranquillité relative des habitants pour s'imposer, il faut aussi s'en faire aimer pour qu'ils acceptent leur domination. Les habits du sauveur sont un costume impeccable en la matière. Les mises en scène comme les occasions d'intervenir sont nombreuses : on se souvient, à la Rocinha, de la construction d'une salle de concert tout équipée financée par Lulu, jeune dono mort depuis ; on évoque l'aide financière apportée aux familles les plus pauvres, le paiement du médecin et des médicaments quand l'argent vient à manquer, la télévision volée et rapportée, l'organisation de fêtes et de concerts mémorables... et puis le collège. $\mathrm{Ce}$ 
collège qui occupe une place centrale dans la vie de nombreux habitants de la communidade et qui doit surtout offrir des perspectives d'avenir meilleur aux adolescents qui y sont scolarisés. Le dono et ses lieutenants se font fort alors de prendre le parti de la protection de cet espace particulier dans lequel ils ne pénètrent pas, mais dont ils se font les défenseurs. La séquestration du patron d'une société de minibus a marqué les esprits : il a été libéré à la condition d'assurer gratuitement le transport de tous les élèves de la favela jusqu'à leurs écoles. Certains élèves expliquent qu'une partie des équipements de foot de l'établissement ont été payés par le dono, d'autres assurent que les cahiers et les livres viennent des mêmes fonds. Autant d'informations que la direction du collège a formellement démenties, même s'il semble, d'après certains enseignants, que l'association des habitants, vitrine légale des trafiquants, a bien pu participer occasionnellement au financement de quelques actions. Mais les formes de pression sur l'école sont souvent plus subtiles. Les enseignants et la direction se défendent d'ailleurs d'avoir quoi que ce soit à voir avec les bandidos do trafico. Nos questions pour essayer de comprendre cette tolérance des trafiquants vis-à-vis d'une structure qui leur échappe nous a valu des réponses évasives et parfois gênées. Entendons-nous bien : il ne s'agit pas de dire que les membres de la communauté éducative ont négocié avec les trafiquants un pacte de non-agression ou leur tranquillité. Il ne semble pas y avoir de contacts directs entre les trafiquants et les enseignants, mais ces derniers ne sont pas dupes des bénéfices de cette neutralité bienveillante.

La protection de l'école est donc une habitude que les élèves pour leur part connaissent parfaitement. Joca nous explique un jour, à propos d'un véhicule de police constamment stationné à proximité de l'entrée de l'établissement, que «ça ne sert à rien ! Le patrão protège le collège, personne ne peut rien y faire ! Si quelqu'un rentre pour faire le bazar, ça peut aller très très mal pour lui. Il est sûr qu'il sera sévèrement frappé, peut-être même tué. Le collège, c'est trop important pour la communidade. Le premier qui y touche, il est foutu, c'est sûr. Même si t'es élève et qu'il apprend que tu perturbes la classe ou les activités, tu peux passer un mauvais quart d'heure. Moi il m'est arrivé d'aller taper d'autres élèves parce qu'ils faisaient n'importe quoi. Ça ne se fait pas. Dehors oui, mais pas ici ${ }^{9} »$. Le collège apparaît donc clairement comme un espace protégé par le dono et par ailleurs très apprécié de la communidade.

Il ne faut donc pas céder à une vision romantique selon laquelle l'ouverture du collège, la mise en partenariat ou la diversification des actions qui y sont menées constitueraient la clé de tous les maux liés à la violence à l'école, ou plus généralement une potion

\author{
Échange \\ avec un professeur \\ de portugais \\ Nous parlons \\ depuis un moment \\ lorsque je lui demande \\ s'il ne trouve pas bizarre \\ que le collège n'ait jamais \\ de problème \\ avec les trafiquants, \\ ni même avec les élèves \\ enrôlés dans le trafic. \\ Il me répond \\ avec un sourire gêné que \\ « ce doit être parce que \\ le collège est en bordure \\ de la favela et ne gêne pas \\ le trafic ». Je me montre \\ peu convaincu \\ par sa réponse \\ et lui demande s'il n'y aurait \\ pas des raisons \\ plus profondes. \\ Il sourit toujours et me dit \\ qu'il ne sait pas trop, \\ qu'il ne se pose pas \\ ces questions. \\ Il ajoute quand même \\ qu'il sait que les donos \\ de la Rocinha ont toujours \\ protégé les écoles, \\ que ça fait partie \\ de leur image \\ et de leur réputation \\ (extrait du journal de bord, \\ 8 juillet 2004).
}

9. Extrait du journal de bord. 


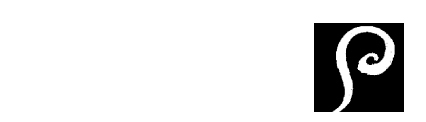

La violence à l'école

L'objectif de l'école

n'est donc pas

de transmettre un savoir,

mais plutôt de fournir

un environnement scolaire

susceptible de pallier une socialisation défaillante. magique pour assurer la construction d'un rapport au savoir et à l'école moins conflictuel dans les quartiers populaires. Sans doute l'adhésion de la population et de nombreux élèves à l'exercice scolaire comme aux activités plus larges qui y sont proposées est sincère et représente en soi un intérêt particulier. En ce sens, si le dono maintient sur les élèves une certaine pression, les propos de ces derniers comme ceux de leurs enseignants témoignent d'un attachement à l'école qui la dépasse largement. Elle révèle déjà la pertinence d'une ouverture du monde scolaire pour construire un lien social qui ne se situe pas toujours sur le registre de la domination et qui permet aussi de donner peut-être un sens plus large et salutaire à la mission de l'école. Mais elle n'est pas ici la seule raison du calme et de la sérénité de cet établissement. Le collège est aussi un instrument de pouvoir à la Rocinha, sa tranquillité participe indirectement à la construction de l'oppression paternaliste et nécessite donc d'être protégée. Il s'agit donc d'un calme relatif, acquis au prix d'une connivence avec le monde de la rue qui, si elle n'est pas vraiment assumée, existe incontestablement.

\section{ÉDUQUER PLUTÔT QU'APPRENDRE : LE CHOIX BRÉSILIEN}

Les modes de gestion de la confrontation entre les normes scolaires et les normes juvéniles sont au cœur des préoccupations des enseignants brésiliens. Mais ils appellent à des stratégies d'évitement plus qu'à une confrontation directe avec les élèves, révélant des différences fondamentales dans la conception même de l'école entre le Brésil et la France par exemple.

L'immense majorité des enseignants que nous avons côtoyés revendique une mission qui est d'abord celle d'éduquer les adolescents. C'est là une approche très différente du modèle républicain français, particulièrement attaché à une forme scolaire dans laquelle les adolescents sont d'abord des élèves, censés être égaux devant les apprentissages et les possibilités de réussite. Pour les enseignants brésiliens, les élèves sont d'abord des enfants pauvres qui souffrent d'un manque d'éducation qu'il faut compenser.

Ainsi les formes scolaires organisées exclusivement autour des apprentissages semblent peu pertinentes puisqu'elles font courir le risque, d'après les professeurs, d'un absentéisme qu'ils redoutent par-dessus tout. C'est ainsi que des «aménagements » sont proposés pour certains élèves faisant l'objet d'attentions particulières qui visent à ne pas prononcer leur rupture avec le collège. Joca, dont nous avons parlé, chef de l'un des gangs liés au trafic, était ainsi absent à toutes les évaluations comme à certains cours sans que ça ne lui soit jamais reproché. Il a même continué d'être admis dans les niveaux supérieurs au fur et à mesure de sa scola- 
rité secondaire. Le collège est donc envisagé comme un espace qui est d'abord un lieu d'épanouissement pour les adolescents, une sorte de refuge offrant un peu de sérénité dans un environnement perçu comme dangereux. Cette situation explique sans doute en partie le mauvais classement du Brésil dans les enquêtes internationales sur la qualité des apprentissages. Même si ces classements sont sujets à discussion ${ }^{10}$, il est intéressant de noter que le Brésil est classé $53^{\mathrm{e}}$ sur les compétences en mathématiques (sur 57 pays) et $48^{\mathrm{e}}$ en lecture (sur 56 pays ${ }^{11}$ ).

L'exigence scolaire dans les écoles publiques, en termes d'apprentissages, est ainsi souvent dépassée par un impératif de protection et de socialisation que les enseignants jugent prioritaire. Les apprentissages ne sont pas pensés comme un moyen d'atteindre un tel but (Rochex, 1998), mais comme une source de violence symbolique qu'il est préférable d'éviter. Bien sûr, le manque d'exigence scolaire ne suffit pas à expliquer les faibles niveaux de violence à l'école au Brésil. À l'inverse, il serait abusif de conclure que les difficultés que rencontrent la France et d'autres pays européens en la matière sont dues au seul fait d'une doxa scolaire plus contraignante. Mais la nature comme la forme de l'enseignement proposé contribuent à développer une forme de tension qui est de toute manière constitutive de l'acte pédagogique (Charlot, 1997). La question est donc de savoir dans quelle mesure et selon quelles modalités il est possible de composer avec cette tension, de la contenir, pour que l'acte pédagogique conserve sa dimension symbolique. L'école brésilienne publique fait aujourd'hui le choix de l'esquive, mais perd ainsi tout crédit dans sa volonté de devenir un moyen de mobilité sociale. L'école française, plus que d'autres systèmes européens comparables d'ailleurs, fait celui d'une forme de sanctuarisation de l'espace scolaire, qui alimente les attitudes d'opposition en oubliant que l'environnement immédiat de l'école aussi peut être une ressource. Il est donc nécessaire de trouver un équilibre entre ouverture sociale ouverte et exigence académique, conditions nécessaires de l'avènement d'une école qui, si elle peut protéger, doit aussi répondre à l'impératif démocratique que suppose une école émancipatrice. Un défi qui reste aujourd'hui à relever.

\section{Biblographie}

BALLION, R. 2000. Les conduites déviantes des lycéens, Paris, Hachette. BENBENISTHY, R. ; AstOR, R. A. 2005. School Violence in Context: Culture, Neighborhood, Family, School and Gender, New York, Oxford University Press. BLAYA, C. 2006. Violences et maltraitances en milieu scolaire, Paris, Armand Colin. ChARLOT, B. 1997. Du rapport au savoir : éléments pour une théorie, Paris, Anthropos. DEBARBIEUX, É. 1999. La violence en milieu scolaire. Tome 2 : Le désordre des choses, Paris, ESF.
10. Cf. à ce sujet

le numéro

de La revue française

de pédagogie, «PISA : analyses

secondaires, questions et

débats

théoriques et

méthodologiques ",

2007, 157.

11. Enquête PISA 2006 portant sur des pays de l'OCDE et des pays partenaires dont le Brésil : http://www.oecd.org. 
La violence à l'école

\section{Mots-clés :}

Violence à l'école, savoirs, comparaison internationale, environnement scolaire.

\section{Key words :}

School violence, knowledge, international comparison, school in context.
DeBARBIEUX, É. 2006a. "La violence à l'école : quelques orientations pour un débat scientifique mondial », International Journal on Violence and School, 1, 18-28. DeBARBIEUX, É. 2006b. Violence à l'école : un défi mondial?, Paris, Armand Colin. GefFraY, C. 1995. Chroniques de la servitude en Amazonie brésilienne : essai sur l'exploitation paternaliste, Paris, Karthala.

GOTTFREDSON, D. C. 2001. Schools and delinquency, Cambridge, Cambridge University Press.

Ireland, V. ; Charlot, B. ; Gomes, C. ; Gusso, D. ; De Carvalho, L. C. R. ; Fernandes, M. ; ENNAFAA, R. ; GARCIA, W. 2007. Repensando a escola : um estudo sobre desafios de aprender, ler e escrever, Brasilia, Inep, Unesco.

MISSE, M. 2008. « Le movimento. Les rapports complexes entre trafic, police et favelas à Rio de Janeiro ", Déviance et Société, 32, 495-506.

MoIGNARD, B. 2007. " Le collège comme espace de structuration des bandes

d'adolescents dans les quartiers populaires : le poids de la ségrégation scolaire", Revue française de pédagogie, 158, 31-42.

MoIGNARD, B. 2008. L'école et la rue : fabriques de délinquance, Paris, PUF.

OZBolt, B. ; PEYRAT, S. 2007. La guerre des normes, Paris, L'Harmattan.

RIO CALDEIRA, T. P. (do) 2002. Cidade de muros. Crime, segregação e cidadania em São Paulo, São Paulo, Editora 34, Edusp.

ROCHEX, J.-Y. 1998. Le sens de l'expérience scolaire, Paris, PUF.

\section{RÉSUMÉ}

La France est plus touchée que le Brésil par la violence à l'école. Alors que ce pays connaît des niveaux de violence endémique, comment l'école parvient-elle à se préserver ? Quels enseignements tirer de l'expérience brésilienne ? À partir d'une enquête comparative sur le lien entre violence à l'école et environnement scolaire, cet article propose de mettre en perspective les choix que révèle un certain nombre de postures scolaires et leur influence sur l'état de la violence à l'école.

\section{SUMMARY}

France is more affected by school violence than Brazil. How do Brazilian schools manage to remain so well preserved when Brazil is confronted with an endemic level of violence? What can we learn from the Brazilian experience? By the means of a comparative survey on the link between school violence and the scholastic environment, this review attempts to put into perspective the choices revealed by a certain number of academic positions and their influence on the state of violence at school. 